University of Zurich

Department of Economics

Working Paper Series

ISSN 1664-7041 (print)

ISSN 1664-705X (online)

Working Paper No. 55

\title{
Siesta: A theory of freelancing
}

Maria Saez Marti

December 2011 


\title{
Siesta \\ A theory of freelancing
}

\author{
Maria Saez Marti* \\ Department of Economics \\ University of Zurich
}

\begin{abstract}
I study the effect of fatigue and innate ability on performance in a model with incomplete contracts, lumpy tasks requiring multiple periods of work and stochastic productivity shocks. I find that increasing ability or reducing fatigue does not lead necessarily to more productive efficiency, since it may exacerbate the incentive for agents take "too much" on-the-job leisure. In a world with heterogenous agents, the problem may be ameliorated by the introduction of a dual labour market with freelancers (who can take breaks at their discretion) and regular workers (who work on a fixed schedule).
\end{abstract}

JEL classification: D0, D8, D9, M51, M55.

Keywords: Breaks, leisure, productivity, freelancing.

*I thank Florian Engl, Isabella Ferru, Johan Lagerlöf, Frederic Schneider, Fabrizio Zilibotti and Jörgen Weibull for useful comments. 
...continual labor will break the power of our minds.

Seneca

Inspiration does exist, but it must find you working.

Pablo Ruiz Picasso

\section{Introduction}

The terms on-the-job leisure and shirking are sometimes used interchangeably in the economics literature. An aspect which is often neglected is that leisure may increase workers' productivity (Florence (1924), Hamermesh (1990), Mednick et al. (2002), Mednick and Ehrman (2007)). ${ }^{1}$ In this case it would be in the employers' interest to allow workers to take on-the-job resting breaks rather than forcing them to work all the time. ${ }^{2}$ Since neither do all workers get equally tired, nor are all tasks equally tiring, breaks benefit different workers to different extents. Fix timetables result in productive inefficiency since too many, or too few workers, would take breaks, from the employers' viewpoint.

In this paper I develop a principal agent model with unobservable fatigue and stochastic (innate) ability to study the impact of fatigue. This model allows to determine which type of workers should be allowed to take breaks as well as the design of incentive compatible contracts which allow workers' sorting into the right work schedule.

I consider an agent who has a day to finish a task. The task consists of two parts, each requiring a full period of work. The agent can work in the morning, in the afternoon and in the evening. The task has value only if complete and this value can only be realized at the end of the day. The probability of completing a part depends on the worker's innate ability and on how tired he gets. Workers are heterogenous and differ in innate ability and/or fatigue, which are assumed to be separable. I also assume that fatigue is monotonic so that agents who are more tired after one period of work will also be more tired after an additional period of work. These two assumptions allow the ranking of agents in either dimension, making statements like "agent $i$ gets more tired than agent $j$ " or " $i$ is more able than $j$ " meaningful.

Fatigue can be eliminated by taking a break (or siesta) in the middle of the day, but this reduces the number of periods in which the worker can work. Ceteris paribus, agents with higher innate ability are better performers and so are agents who get less tired. When the timing of effort is endogenous it is no longer true that more able and less tired agents are better performers.

I show first that not all workers should be allowed to take breaks. In particular breaks should be taken by all agents if fatigue is strong, but only the most able agents should take breaks when fatigue is mild. If leisure/work has intrinsic value and workers are free to choose

\footnotetext{
${ }^{1}$ Mednick et al. (2002) show that taking a long nap increases performance on a learning task. They let subjects repeat the same visual texture discrimination task four times a day for 60 minutes. Some subjects were allowed to take naps of different length between the second and third repetition of the task. While the performance of those subjects who weren't allowed to take a nap actually decreases over the course of the day, those who took the longest one were as alert as in the morning (when performance was at its highest).

${ }^{2}$ Some firms like Google do provide facilities in the workplace for resting. Pilots of British Airways are encouraged to rest during intercontinental flights.
} 
their timetable and do so to maximize their expected utility there will be some workers who fail to take breaks when they should, and others who will take them when they should not. Only when the opportunity cost of leisure is zero are the interest of the worker and the employer aligned.

Introducing discounting does alter the qualitative predictions of the model but only when the opportunity cost of working is strictly positive. In this case there will be a new type of agent: the one who arrives late to work and fails because of lack of time. This behaviour is utility maximizing only if the agent has high ability and fatigue is weak.

In a standard model with asymmetric information, the employer would like to design an incentive compatible scheme that make workers willing to work rather than shirk (enjoy leisure). This is the case for instance in Shapiro and Stiglitz (1984) where wages above market clearing guarantee that the worker is willing to work hard in order to avoid long spells of unemployment. If workers' fatigue cannot be observed by the principal and leisure not only increases the worker's utility but also boosts workers' productivity in an unequal manner (because they are effected by fatigue to different extents) a single contract will result in productivity losses. I show that the principal can separate workers by offering contracts which differ in two dimensions: i) the bonus paid for the finished task and ii) the freedom of timetable workers have. If lower bonuses are accompanied by more freedom those workers who should take breaks may decide to be freelancers and enjoy more leisure. An alternative contract with rigid timetable and no leisure opportunities can be made attractive to those workers whose productivity is not much affected by fatigue if the bonus payment compensates for the forgone leisure utility. This incentive compatible dual market results in two groups of workers: freelancers who take breaks at their discretion and office workers who work all the time but get higher wages.

The paper is organized as follows. Section 2 presents the model, section 3 characterizes the behaviour that maximizes the probability of completion of the task. In section 4, I endogenize the timing of effort and analyze the cases in which the interests of the principal and the workers are misaligned; i.e the opportunity cost of working is different from zero. In this section I characterize the probability of completion induced by the utility maximizing agents and show that increasing ability or reducing fatigue not always lead to better performances. In section 5 I introduce a dual market with freelancers and regular workers and show that this allows the principal to achieve perfect sorting and increase productive efficiency. Section 6 concludes.

\section{The model}

Consider an agent who is given a task to be finished by the end of the day. The task consists of two parts and has no value if incomplete. The agent receives a bonus $B$ for the complete task, nothing otherwise. The working day is divided in three periods: morning, afternoon and evening. Each part requires that the agent works on it a whole period. Every time he works on a part there is a positive probability that he completes it; but this probability is affected by fatigue and declines with the number of periods he has worked before. The disutility of working is $e$.

Breaks eliminate fatigue but reduce the number of available periods so that if the agent does not work in the afternoon his productivity in the evening is like the morning's, but he forgoes a 
period of work and his only chance to finish is in the evening. The leisure value of breaks is $u$. The agent has to decide when to work.

Let $\pi_{t} \in[0,1]$ denote the probability of succeeding in the part the agent is working on when he has worked, without any break, $t$ periods before and let $\boldsymbol{\pi}=\left(\pi_{0}, \pi_{1}, \pi_{2}\right)$ be the productivity profile. I will refer to $\pi_{0}$ as the agent's (innate) ability.

The effect of fatigue is captured by a parameter $\alpha \in[0,1]$. The larger $\alpha$, the less the agent's productivity is affected by previous efforts. In particular when $\alpha=1, \pi_{t}=\pi_{0}$ for all $t$, when $\alpha=0, \pi_{t}=0$ for $t=1,2$. Any intermediate value of $\alpha$ leads to a declining productivity profile; i.e $\pi_{t+1}<\pi_{t}$. I assume that innate productivity and fatigue are separable, and that the latter is monotonic:

ASSUMPTION 1 (Separability). The productivity vector $\boldsymbol{\pi}$ can be written as,

$$
\boldsymbol{\pi}\left(\pi_{0}, \alpha\right)=\pi_{0}(1, \alpha, f(\alpha)),
$$

with $f(\alpha) \leq \alpha$.

ASSUMPTION 2 (Monotonicity). Fatigue is monotonic if

$$
\operatorname{sign}\left(\alpha-\alpha^{\prime}\right)=\operatorname{sign}\left(f(\alpha)-f\left(\alpha^{\prime}\right)\right)
$$

for any $\alpha$ and $\alpha^{\prime}$.

Hereafter I assume that $f:[0,1] \rightarrow[0,1]$ is a twice continuously differentiable increasing function with $f(0)=0$ and $f(1)=1$ and that all agents have the same fatigue function $f$. These assumptions allow for the ranking of agents on the fatigue dimension and on the ability one. I will say that agent $i$ gets more, less or equally tired than agent $j$ whenever $i$ 's fatigue parameter $\alpha$ is smaller, equal or larger than $j$ 's. Similarly, I will say that agent $i$ is more, less or equally able than agent $j$ whenever $i$ 's initial ability is larger, smaller or equal than $j$ 's. Agent $i$ will be described by the vector $\pi^{i}$.

The probability of completion depends on the timing of effort which in turn depends on the fatigue, the ability and the other parameters of the model, $u, e$ and $B$. In particular the ratio between the opportunity cost of working and the bonus will play a key role. Let $v$ denote such ratio:

$$
v=\frac{u+e}{B}
$$

I assume that the agent chooses his timing of effort to maximize his expected discounted utility. Discount is exponential with discount factor $\delta$. I first analyze the benchmark case in which the agent chooses the timing that maximizes the probability of completion. I then study the utility maximizing behaviour and see how it departures from the benchmark.

\section{Maximizing the probability of completion}

Consider first the point of view of the principal who is interested in the agent completing the task with the highest probability. Abstracting from its leisure value, ${ }^{3}$ having an afternoon break

\footnotetext{
${ }^{3}$ Most likely not internalized by the principal.
} 
will only be optimal if the morning was productive, i.e inspiration arrived and found the agent working. Moreover, working in the evening is only worthwhile if the agent is, by then, half way through.

Suppose that the agent does not plan a break and works every period until he has the whole thing, or he has to call it a day. The probability of succeeding under this work plan, $\Pi^{w}(\boldsymbol{\pi})$, is ${ }^{4}$

$$
\Pi^{w}(\boldsymbol{\pi})=\pi_{0} \pi_{1}+\pi_{0}\left(1-\pi_{1}\right) \pi_{2}+\left(1-\pi_{0}\right) \pi_{1} \pi_{2}
$$

The probability of succeeding if he takes a break after a productive morning, $\Pi^{b}(\boldsymbol{\pi})$, is

$$
\Pi^{b}(\boldsymbol{\pi})=\pi_{0}^{2}+\left(1-\pi_{0}\right) \pi_{1} \pi_{2} .
$$

In order to maximize the probability of completion he should have a break whenever the difference between (5) and (4), $\Delta(\boldsymbol{\pi})$, is non-negative:

$$
\Delta(\boldsymbol{\pi})=\pi_{0}\left(\pi_{0}-\left(\pi_{1}+\left(1-\pi_{1}\right) \pi_{2}\right) \geq 0 .\right.
$$

This will be the case when

$$
\pi_{0} \geq \pi_{1}+\left(1-\pi_{1}\right) \pi_{2} .
$$

It is easy to see that (7) holds whenever $\pi_{0}=1$. In this case a break guarantees completion while continual labour leads to failure with positive probability. The breaking condition can be written, after substituting (1) in (7), as:

$$
1 \geq \alpha+\left(1-\pi_{0} \alpha\right) f(\alpha)
$$

Note that an agent should take a break whenever

$$
\pi_{0} \geq \frac{\alpha+f(\alpha)-1}{\alpha f(\alpha)} \equiv \pi_{b}(\alpha)
$$

and that $\pi_{b}(\alpha)$ is increasing in $\alpha$ and positive only when $\alpha+f(\alpha)>1$. Note that all agents should take a break when $\alpha+f(\alpha) \leq 1$. The following lemma is straightforward implication of $(9)$.

LEMMA 1. Agent $i$ will take a break whenever (7) holds for $\boldsymbol{\pi}^{i}$. If agent $i$ should take a break, then so should any agent who is equally able but gets more tired and any agent who gets equally tired but is more able.

Fatigue monotonicity implies that when two agents who are equally able but differ in their fatigue choose the same timing of effort, the one who gets less tired will finish with higher probability. Similarly, an agent who is more able will finish with a higher probability than another agent who is less able and gets equally tired, provided they both choose the same timing of effort. This observation leads to the following result.

\footnotetext{
${ }^{4}$ For simplicity I will write all the expressions using the $\pi_{t}$ 's and later substitute (1) in and use $\pi_{0}$ and $\alpha$ as arguments instead of $\boldsymbol{\pi}\left(\pi_{0}, \alpha\right)$.
} 
PROPOSITION 1. The maximum probability of completion, $\Pi^{*}\left(\pi_{0}, \alpha\right)$,

$$
\Pi^{*}\left(\pi_{0}, \alpha\right)=\max \left\{\Pi^{b}\left(\boldsymbol{\pi}\left(\pi_{0}, \alpha\right)\right), \Pi^{w}\left(\boldsymbol{\pi}\left(\pi_{0}, \alpha\right)\right)\right\},
$$

is monotonically increasing in $\alpha$ and in $\pi_{0}$.

Proof: Both $\Pi^{b}\left(\boldsymbol{\pi}\left(\pi_{0}, \alpha\right)\right)$ and $\Pi^{w}\left(\boldsymbol{\pi}\left(\pi_{0}, \alpha\right)\right)$ are increasing in $\alpha$ and $\pi_{0}$.

Example (Exponential fatigue): Exponential fatigue, $\pi_{t}=\alpha^{t} \pi_{0}(\alpha \leq 1)$, satisfies fatigue monotonicity. Equations (4), (5) and (6) can be written as

$$
\begin{gathered}
\Pi^{w}(\boldsymbol{\pi})=\pi_{0}^{2}\left(\alpha+\alpha^{2}\left(1-\alpha \pi_{0}\right)+\alpha^{3}\left(1-\pi_{0}\right)\right), \\
\Pi^{b}(\boldsymbol{\pi})=\pi_{0}^{2}\left(1+\alpha^{3}\left(1-\pi_{0}\right)\right)
\end{gathered}
$$

and

$$
\Delta(\boldsymbol{\pi})=\alpha^{3} \pi_{0}^{2}\left(\pi_{0}-\pi_{b}(\alpha)\right)
$$

where

$$
\pi_{b}(\alpha)=\frac{1}{\alpha}\left(1+\frac{1}{\alpha}-\frac{1}{\alpha^{2}}\right) .
$$

Note that (14) is increasing in $(0,1), \pi(1 / \varphi)=0$ and $\pi(1)=1$, where $\varphi$ is the golden ratio, i.e $(1+\sqrt{5}) / 2 .^{5}$ For values of $\alpha \leq 1 / \varphi$ a break is always productivity enhancing.

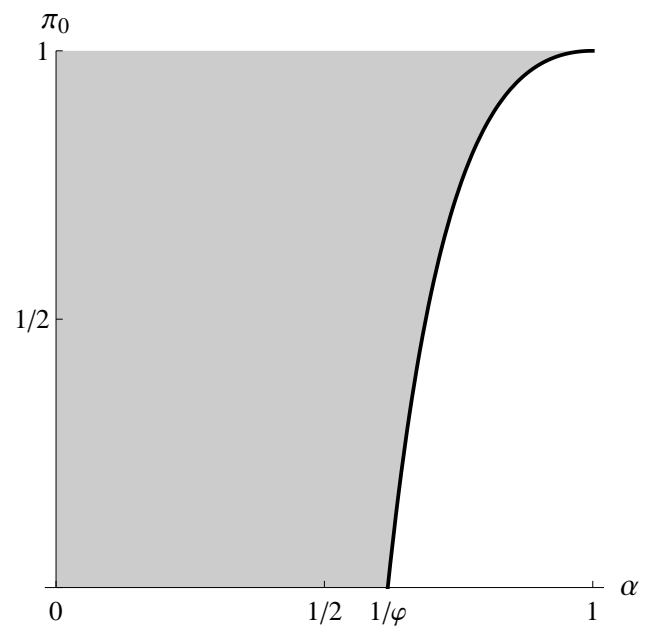

Figure 1: $\Delta(\boldsymbol{\pi}), \pi_{t}=\alpha^{t} \pi_{0}$.

Figure 1 partitions the space $\left(\alpha, \pi_{0}\right)$ in two areas, the gray one corresponds to those parameter values for which a break increases the probability of completion and the white to those for which the break has the opposite effect. The thick line corresponds to the pairs $\left(\alpha, \pi_{0}\right)$ for which

\footnotetext{
${ }^{5}$ I thank Isabella Ferru for pointing this out.
} 
$\Delta(\boldsymbol{\pi})=0$, i.e, to $\left(\alpha, \pi_{b}(\alpha)\right)$. Note that for all $\alpha<1 / \varphi$ all agents, irrespective of productivity, should take a break. For values above $1 / \varphi$ only the most productive workers should break. As $\alpha$ increases beyond $1 / \varphi$, the range of initial productivities for which a break is optimal, $\left[\pi_{b}(\alpha), 1\right]$ shrinks.

Figure 2 shows $\Pi^{w}$ (solid) and $\Pi^{b}$ (dashed) as functions of $\alpha$ for a given $\pi_{0}>0$. Note that for all $\alpha>\hat{\alpha}$ a break leads to a lower probability of completion. When $\alpha=0$ the only way to finish is by taking a break, the probability of completion being $\pi_{0}^{2}$.

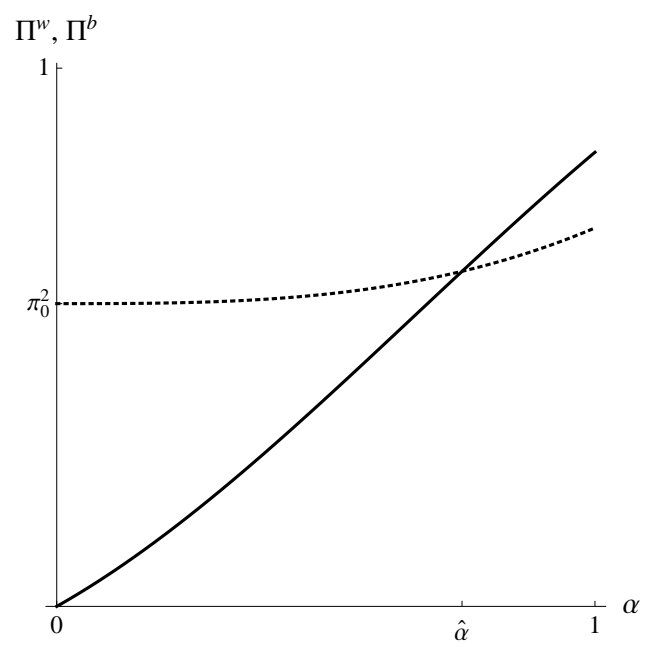

Figure 2: $\pi_{t}=\alpha^{t} \pi_{0}$.

When the opportunity cost of work is different from zero, some utility maximizing agents will choose breaks in a way that does not maximize the probability of completion, some may give up (i.e no to re-start after an unsuccessful morning) and some may decide to start late (i.e in the afternoon). These behaviours will never be observed if $v=0$. In this case a utility maximizing agent behaves "as if" he maximized the probability of completion.

In the following section I assume that $v \neq 0$ and derive a set of indifference conditions determining which agents will be indifferent between taking breaks or not, between giving up or not and between starting in the morning or in the afternoon. This allows the partition of the $\left(\alpha, \pi_{0}\right)$ space into regions where behaviour is constant and to analyze how this behaviour changes with fatigue and innate ability. This will be done in lemmata 2, 3 and 4 . The endogenous probability of completion resulting form the utility maximizing behaviour is shown to be piecewise non decreasing (proposition 3). The reason for this result is that moving from one region into another leads to discrete jumps in the probability of completion, but, in a given region, since the timing is constant, reducing fatigue (increasing $\alpha$ ) or increasing ability (increasing $\pi_{0}$ ) is never accompanied by lower probabilities of completion. I will then analyze the probability of completion and pin down the configurations for which decreasing fatigue or increasing ability lead to lower rates of success (propositions 4 and 5). 


\section{The value of breaks}

Let $B, u$ and $e$ be the bonus for finishing the task, the utility derived from the break and the disutility of work, respectively. Since my objective is to study the effect fatigue, productivity and discounting have on the timing of effort, I shall assume hereafter that the parameters are such that the task is worth being undertaken by a perfectly patient agent even if the effect of fatigue is extreme, i.e, $\pi_{1}=0$. In particular, I assume that

$$
-e+\pi_{0}\left(\delta u-\delta^{2} e+\pi_{0} \delta^{3} B\right)+\left(1-\pi_{0}\right)\left(\delta+\delta^{2}\right) u>u\left(1+\delta+\delta^{2}\right)
$$

holds for $\delta=1$, where $\delta$ is the discount factor. Substituting $\delta=1$ in (15) and rearranging I obtain,

$$
v<\frac{\pi_{0}^{2}}{1+\pi_{0}} \in[0,1 / 2]
$$

where $v=(u+e) / B$ is the opportunity cost of working relative to the value of the task.

Time spent away from the task results in a leisure utility gain and increases the productivity on the task in the future if the break happens to take place in the afternoon.

Consider an agent who arrives to the last period having completed half task. He will want to complete if

$$
-e+\pi_{t} \delta B>u
$$

where $\pi_{t}$ takes values $\pi_{0}$ if the agent had a break before, $\pi_{2}$ if he started in the morning and did not have the break and $\pi_{1}$ if he started late, i.e in the afternoon. Condition (17) can be re-written as

$$
\pi_{t} \delta>v
$$

Assume that (18) holds for $t=2$, the morning is over and the agent has successfully finished half task now it is time for a break. After the break he will be as fresh as in the morning, ready to attempt the second part. Having a break now gives immediate utility $u$, postpones for sure the disutility of working $e$ to the evening and increases the probability of finishing then to the morning's value $\pi_{0}$. The present discounted value of the afternoon break, taken after a successful morning, is

$$
u-\delta e+\delta^{2} \pi_{0} B
$$

Skipping the break anticipates the disutility of working to now and increases the probability of not having to work on the onerous task in the evening and enjoying leisure then. The probability of completing the task in the afternoon is increased at the expenses of the evening's. These trade-offs lead to the following present discounted value,

$$
-e+\delta\left(\pi_{1} u-\left(1-\pi_{1}\right) e\right)+\delta^{2}\left(\pi_{1}+\left(1-\pi_{1}\right) \pi_{2}\right) B .
$$

The agent will be indifferent between the two alternatives when

$$
(u+e)\left(1-\pi_{1} \delta\right)=\delta^{2} B\left(\pi_{1}+\left(1-\pi_{1}\right) \pi_{2}-\pi_{0}\right) .
$$

The left hand side is the change in the leisure utility due to the early break. This utility 
includes the direct break's utility and the forgone disutility of work. The right hand side is the change in the expected value of the task due to the change in the probability of completion. Equation (21) can be re-written as

$$
v=\frac{\delta^{2}\left(\pi_{1}+\left(1-\pi_{1}\right) \pi_{2}-\pi_{0}\right)}{1-\pi_{1} \delta}=\frac{-\delta^{2} \Delta(\boldsymbol{\pi})}{\pi_{0}\left(1-\pi_{1} \delta\right)} .
$$

The function

$$
v_{b}(\boldsymbol{\pi}, \delta)=\frac{\delta^{2}\left(\pi_{1}+\left(1-\pi_{1}\right) \pi_{2}-\pi_{0}\right)}{1-\pi_{1} \delta}
$$

gives, for each vector of probabilities $\pi$ and discount factor $\delta$, the value of $v$ that makes the agent indifferent between taking an afternoon break and continuing working. The agent will want to take a break rather than continue (conditional on a successful morning) whenever

$$
v \geq v_{b}(\boldsymbol{\pi}, \delta)
$$

LEMMA 2 (BREAKS). Agent $i$ will take a break whenever $v \geq v_{b}\left(\boldsymbol{\pi}^{i}, \delta\right)$. If agent $i$ wants to take a break then so does any agent who is equally able but gets more tired. If agent $i$ wants to take a break then so does any agent who is more able and gets equally tired, provided $\alpha+f(\alpha)<1$. For all $\alpha$ such that $\alpha+f(\alpha)>1$ and small enough $v$ there exists an interval $(\underline{\pi}(\alpha, \delta, v), \bar{\pi}(\alpha, \delta, v))$ with $\bar{\pi}(\alpha, \delta, v)<\pi_{b}(\alpha)$, such that all agents with $\pi_{0} \in(\underline{\pi}(\alpha, \delta, v), \bar{\pi}(\alpha, \delta, v))$ prefer not to take a break. All other agents prefer the break.

Proof: Substituting (1) in (22) we get,

$$
v_{b}\left(\pi_{0}, \alpha, \delta\right)=\frac{\delta^{2} \pi_{0}\left(\alpha+\left(1-\pi_{0} \alpha\right) f(\alpha)-1\right)}{1-\pi_{0} \alpha \delta}
$$

which is increasing in $\alpha$. $v_{b}\left(\pi_{0}, \alpha, \delta\right)$ is decreasing in $\pi_{0}$ whenever the numerator is negative. Assume that $\alpha+f(\alpha)>1$, an agent will be indifferent between taking a break or not when

$$
v\left(1-\delta \alpha \pi_{0}\right)=\delta^{2} \pi_{0}\left(\alpha+\left(1-\alpha \pi_{0}\right) f(\alpha)-1\right)
$$

The LHS is positive for all $v>0$ and linearly decreasing in $\pi_{0}$. The RHS is concave in $\pi_{0}$, takes value 0 at 0 and $\pi_{b}(\alpha)$, reaches a maximum at $\pi_{b}(\alpha) / 2$ and is negative at $\pi_{0}=1$ for $\alpha<1$. For positive but small enough $v$, there are two values of $\pi_{0}, \underline{\pi}(\alpha, \delta, v)$ and $\left.\bar{\pi}(\alpha, \delta, v)\right)$, which solve (26).

Example: $\pi_{t}=\alpha^{t} \pi_{0}$. In this case

$$
v_{b}\left(\pi_{0}, \alpha, \delta\right)=-\frac{\delta^{2} \alpha^{3} \pi_{0}}{1-\pi_{0} \alpha \delta}\left(\pi_{0}-\pi_{b}(\alpha)\right)
$$

Figure 3 shows the contour-map of $v(\boldsymbol{\pi}, \delta)$ in the space $\left(\alpha, \pi_{0}\right)$, the solid lines correspond to a $\delta=1$ and the dashed ones to $\delta<1$. It is easy to see that $v(\boldsymbol{\pi}, \delta)$ takes i) value zero at all pairs $\left(\alpha, \pi_{b}(\alpha)\right)$, ii) negative values where the break has a positive effect on productivity and iii) positive ones when the break results in a fall of the probability of completion. For instance if $v=-0.2$ all agents in the gray area between the contour line corresponding to -0.2 and the 


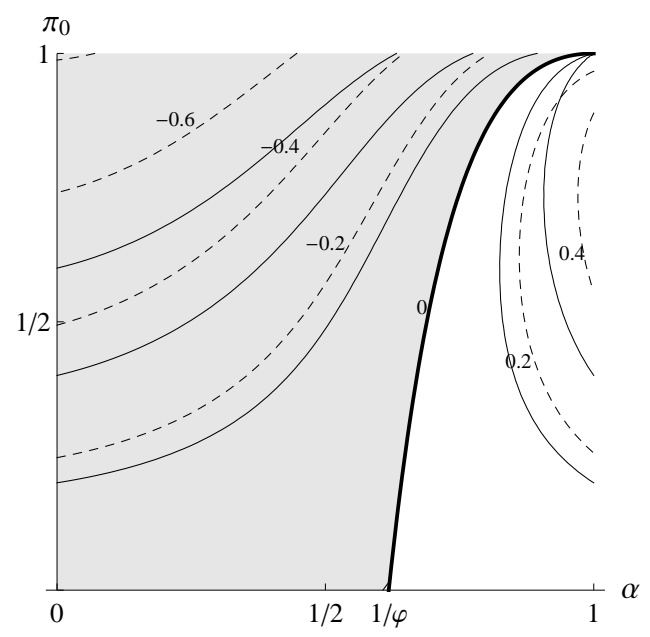

Figure 3: $v_{b}(\boldsymbol{\pi} ; \delta), \pi_{t}=\alpha^{t} \pi_{0}$.

thicker line corresponding to $\left(\alpha, \pi_{b}(\alpha)\right)$ will decide not to take breaks even though this reduces the probability of completion (all agent is the gray area should be taking breaks). Similarly if $v=0.2$, all individuals between the locus $\left(\alpha, \pi_{b}(\alpha)\right)$ and the contour line corresponding to 0.2 will take breaks when they should not (all agent is the white area should not take breaks).

Note that in the white area the contours levels are upward bending: given fatigue, agents with high enough and low enough $\pi_{0}$ will want to have a break for some $v>0$, while those with intermediate $\pi_{0}$ will not. The reasons why the observed behaviour is similar for high and low $\pi_{0}$ is different. Those with low $\pi_{0}$ take the break because were they not to take it they would be very unlikely to finish in the afternoon and would have to work anyway in the evening, forgoing leisure in both periods. By taking the break they enjoy leisure for sure now. Those with high $\pi_{0}$ are very likely to finish anyway and prefer to enjoy leisure now and work later.

When $v<0$ some agents will work too much, not taking a break when they should. This implies that some agents who get more tired and do take breaks finish with a higher probability than equally able agents who get less tired. Similarly some very able agents will work too little, taking a break when they should not. This implies that some agents who are less able but get equally tired will finish with a higher probability. This will never be the case when $v<0$.

Taking a break because of productivity reasons makes sense only when the morning was successful. When this is not the case some agents may decide, if the relative value of leisure is high enough, to give up. I look now at this case, and assume that the morning was unsuccessful. An agent will persevere and start again in the afternoon whenever

$$
-e+\pi_{1}\left(-e \delta+\pi_{2} \delta^{2} B\right)+\left(1-\pi_{1}\right) \delta u \geq u+\delta u
$$

which can be written as

$$
v \leq \frac{\delta^{2} \pi_{1} \pi_{2}}{1+\pi_{1} \delta} \equiv v_{p}\left(\boldsymbol{\pi}^{i}, \delta\right) .
$$


Starting anew makes only sense if the agent is willing to finish a period after, i.e (18) holds for $i=2$. It is easy to see that this is the case whenever (29) holds. As was to be expected those agents whose productivity is most reduced by working are more likely to give up, and so are the impatient agents.

LEMMA 3 (GIVING UP). Agent $i$ will give up whenever $v \geq v_{p}\left(\boldsymbol{\pi}^{i}, \delta\right)$. If agent $i$ wants to give up then so does any agent who is equally able but gets more tired and any agent who gets equally tired but is less able.

Proof: $v_{p}\left(\boldsymbol{\pi}\left(\pi_{0}, \alpha\right), \delta\right)$ is increasing in $\alpha$ and in $\pi_{0}$.

This lemma guarantees that reducing fatigue or increasing ability never affects negatively the probability of completion on the "giving up" margin. As we will see next this is not always true when the decision concerned is starting in the afternoon rather than in the morning.

Starting in the morning does not necessarily maximize the agent's expected utility. For some parameter configurations it might be better to be a late starter and work in the afternoon at the earliest or not to work at all and enjoy leisure in all three periods. This will always be the case if the agent is impatient enough.

Starting in the afternoon is dominated by starting earlier when the agent is patient. Starting in the morning gives, at least, an expected payoff equal to

$$
-e+\pi_{0}\left(\delta u-\delta^{2} e+\pi_{0} \delta^{3} B\right)+\left(1-\pi_{0}\right) u\left(\delta+\delta^{2}\right)
$$

while postponing start leads to a payoff equal to

$$
u-e\left(\delta+\pi_{0} \delta^{2}\right)+\left(1-\pi_{0}\right) \delta^{2} u+\pi_{0} \pi_{1} \delta^{3} B .
$$

Early start (with break and giving up) is preferred to late start whenever

$$
\delta^{3} \pi_{0}\left(\pi_{0}-\pi_{1}\right)>(1-\delta) v
$$

which can be written, after substituting (1), as

$$
\delta^{3} \pi_{0}^{2}(1-\alpha)>(1-\delta) v .
$$

It is easy to see that the LHS of (33) is strictly larger than its RHS whenever $\alpha<1$ and $\delta=1$. Note that (33) is easier violated the larger is $\alpha$, so that agents for whom fatigue is weak are more likely to start late. This leads to the unexpected result that agents who get more tired may out-perform those with less fatigue, even if $v>0$. In order to see this assume that $\delta^{3}>(1-\delta) v$ and that $\pi_{0}=1$. In this case taking a break dominates any other strategy involving starting in the morning, and this is true with and without discounting. Any agent, irrespective of his fatigue, can guarantee completion by starting in the morning and taking a break. All those agents with $\alpha>1-(1-\delta) \delta^{-3}$ prefer to postpone start even though this leads to failure with probability $(1-\alpha)>1$. These agents are outperformed by agents who get more tired but prefer to start earlier. These agents, enjoy leisure in the afternoon rather than in the morning, and finish with probability 1. 
It can also be the case that more productive agents are outperformed by less productive ones who prefer to enjoy leisure later and finish with higher probability. In order to see this assume that fatigue is weak so that agents, when they start in the morning, do not want to give up. Late start is preferred whenever

$$
v\left(1-\pi_{0} \delta+\pi_{1} \delta^{2}\left(1-\pi_{0}\right)\right)>\delta^{3}\left(\pi_{0}^{2}+\left(1-\pi_{0}\right) \pi_{1} \pi_{2}-\pi_{0} \pi_{1}\right) .
$$

which can be written, after substituting (1) in, as

$$
v\left(1-\pi_{0} \delta+\pi_{0} \alpha \delta^{2}\left(1-\pi_{0}\right)\right)>\delta^{3} \pi_{0}^{2}\left(1+\left(1-\pi_{0}\right) \alpha f(\alpha)-\alpha\right) .
$$

This condition is satisfied for large $\pi_{0}$ whenever

$$
v(1-\delta)>\delta^{3}(1-\alpha) .
$$

This results are summarize in the following lemma.

LEMMA 4 (STARTING LATE). If $v(1-\delta)>\delta^{3}(1-\alpha)$ there are some agent who prefer to start late. If agent $i$ wants to start late, then some less able agents who get equally tired and some equally able agents who get more tired prefer to start earlier.

In his most famous quote Edison attributed success to an unbalanced combination of hard work (ninety-nine percent) and inspiration (one percent). Was he right? Are workers who get less tired always better performers? We can now answer these questions in the framework of this simple model.

Let $\Pi\left(\pi_{0}, \alpha, v, \delta\right)$ be the probability of completion of a utility maximizing agent with initial productivity $\pi_{0}$, fatigue $\alpha$, relative value of leisure $v$ and discount factor $\delta$. The following proposition is a straightforward implication of all our previous results.

\section{PROPOSITION 2.}

$$
\Pi\left(\pi_{0}, \alpha, v, \delta\right) \leq \Pi^{*}\left(\pi_{0}, \alpha\right)
$$

with equality for all $\left(\pi_{0}, \alpha\right)$ when $v=0$.

Whenever $v>0$ there are agents who will take breaks and/or will give up, even though this leads to a worse performance. Moreover, if $\delta<1$ some agents may also decide to start late or not to start at all. When $v<0$, some agents will work too long hours at expenses of their productivity. This is probably the case of many academics whose hobby is their work.

As mentioned already, given the same timing more able agents finish with higher probability than less able ones. Similarly, agents who get less tired, keeping timing constant, perform no worse than agents who get more tired. Two agents who only differ in fatigue perform equally well when they choose to take a break and give up. This is summarize in the following proposition.

PROPOSITION 3. Assume that $v \neq 0$. The probability of completion $\Pi\left(\pi_{0}, \alpha, v, \delta\right)$ is piecewise non decreasing in $\alpha$ and in $\pi_{0}$.

Note that the discontinuities occur when there is change in behaviour, and this may lead to downwards jumps in the probability of completion, so that decreasing fatigue (increasing $\alpha$ ) or increasing ability do not lead always to higher probabilities of completion. 
PROPOSITION 4. Assume that agents differ only in fatigue. Then,

i)

$$
\Pi\left(\pi_{0}, \alpha, v, \delta\right) \leq \Pi\left(\pi_{0}, \alpha+\epsilon, v, \delta\right)
$$

for all $\alpha$ and all $\epsilon>0$ whenever $v>0$ and $\delta=1$, and

ii)

$$
\Pi\left(\pi_{0}, \alpha, v, \delta\right)>\Pi\left(\pi_{0}, \alpha+\epsilon, v, \delta\right)
$$

for some $\alpha$ and some $\epsilon>0$ when a) $v<0$ or b) when $v(1-\delta)>(1-\alpha)$ and $\pi_{0}$ is high enough.

Proof: i) i) follows from lemmata 2,3 and 4 . ii) a) let $\alpha\left(\pi_{0}, v, \delta\right)$ solve $v=v_{b}\left(\pi_{0}, \alpha, \delta\right)$, $\alpha\left(\pi_{0}, v, \delta\right)<\alpha\left(\pi_{0}, 0, \delta\right)$ for $v<0$ and agents with $\alpha \in\left(\alpha\left(\pi_{0}, v, \delta\right), \alpha\left(\pi_{0}, 0, \delta\right)\right)$ prefer to work, even though this reduces the probability of completion. ii) b) from lemma 4.

PROPOSITION 5. Assume that agents differ only in innate productivity. Then,

i)

$$
\Pi\left(\pi_{0}, \alpha, v, \delta\right) \leq \Pi\left(\pi_{0}+\epsilon, \alpha, v, \delta\right)
$$

for all $\alpha$ and all $\epsilon>0$ whenever $v<0$ or $\alpha+f(\alpha)<1$, and

ii)

$$
\Pi\left(\pi_{0}, \alpha, v, \delta\right)>\Pi\left(\pi_{0}+\epsilon, \alpha, v, \delta\right)
$$

for some $\pi_{0}$ and some $\epsilon>0$ when $v>0$ and $\alpha$ is large enough.

Proof: Follows from Lemma 2 and 3.

Figure 4 illustrates the two cases in which fatigue can be an advantage (proposition 4,ii)). The solid line in both panels shows the maximum probability of completion as a function of $\alpha$. The dotted lines show how $\Pi\left(\pi_{0}, \alpha, v, \delta\right)$ changes with the fatigue parameter $\alpha$ given $v \neq 0$. The left hand side panel corresponds to $v>0$ and $\delta<1$, the right hand side one to $v<0$. The shaded areas show the loss in productivity due to having an opportunity cost of leisure different from zero. For the positive $v$ 's the losses are due to agents giving up and taking breaks when they should not, or starting late. For the negative $v$ 's the loss is due to agents failing to take productive breaks. It is easy to see that in both panels the probability of completion is piecewise non decreasing with some downwards discontinuities, so that some agents are dominated by others who get more tired. The discontinuity in the left panel is due to the most productive agents postponing start while the others start early and take a break. The latter finish with probability $\pi_{0}^{2}$, the former with the smaller probability $\pi_{0} \pi_{1}$. This is driven by discounting.

Figure 5 illustrates a case in which less able agents outperform more able ones (proposition $5, \mathrm{ii})$ ). The parameters are such that condition (36) is satisfied. The fatigue parameter $\alpha$ is close to 1 (so that the productivity effect of breaks is small), $v>0$ (so that agents tend to take too much leisure) and $\delta<1$ (so that that agents tend to anticipate leisure). It is easy to see that, 

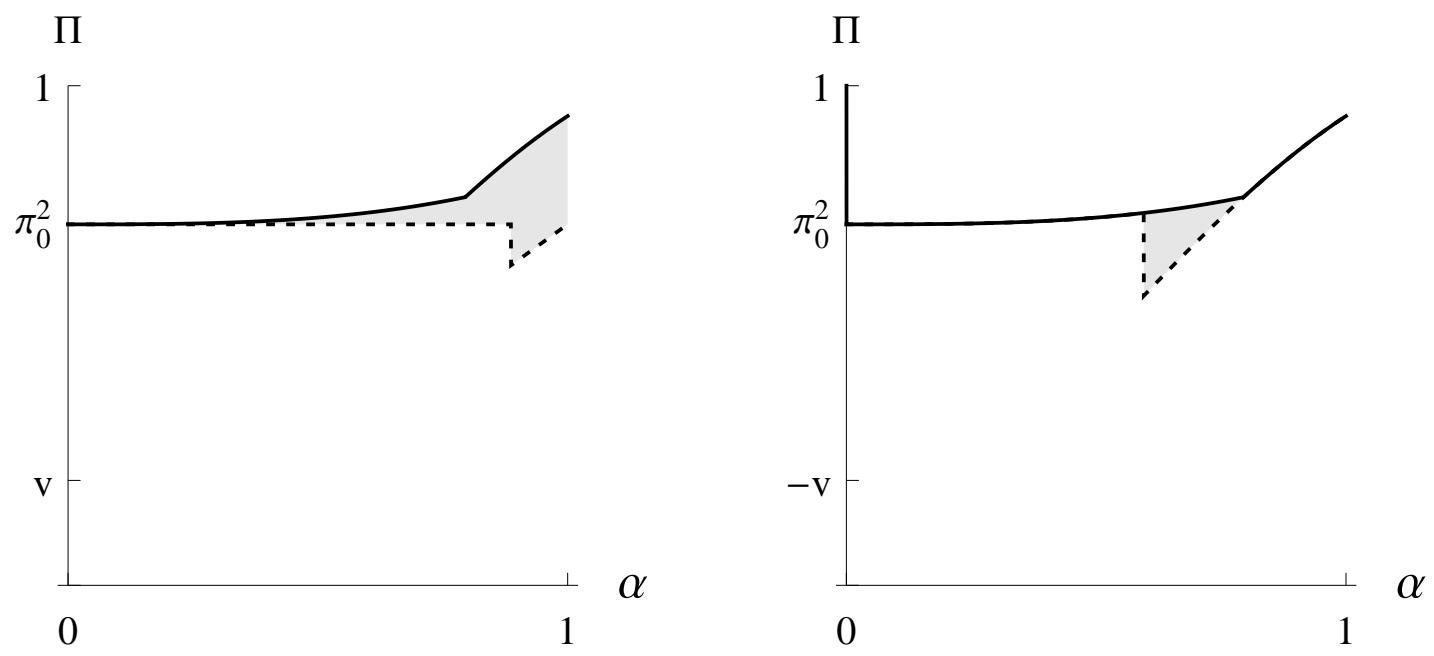

Figure 4: Proposition 4, case ii).

for large $\pi_{0}$ 's, there are three types of agents: $i$ ) those who work all the periods (intermediate levels of $\pi_{0}$ ), $i i$ ) those who start in the morning, do not give up but take a break (even though this leads to less likely completion), so that they can enjoy leisure for sure in the second period and iii) those who start late and enjoy leisure for sure in the morning. The latter are those with very high innate ability and who are pretty sure to finish even though they will only work the last two periods. Note that this type of agent only exist when there is discounting. The last two types may be outperformed by some agent who works all periods even though they are less productive. For small $\pi_{0}$ 's it is better not to start and enjoy leisure all three periods.

So far I have assumed that agents are free to choose their working timetable and decide to take breaks weighing the pros and cons in terms of forgone leisure and changes in the probability of completion. Allowing all workers to take breaks or forbidding all to take them will always result in productivity losses for some workers. Can the principal solve the problem offering different job contracts? In the next section I answer the question. Since the novelty of the paper is to focus on fatigue, I will assume thereafter that all agents have the same innate productivity and that this is observed by the principal. Fatigue, as before, is assumed to be unobservable.

\section{On-the-job leisure}

Assume that $\delta=1$ (so that fatigue is the only force driving the results), $(u+e)>0$ (so that some workers are inclined to take "too much" leisure from the principal' viewpoint) and that all agents are equally able but differ in fatigue. Assume further that there are two types of jobs: i) a freelance one in which the agent is free to choose his work schedule and ii) a regular job 


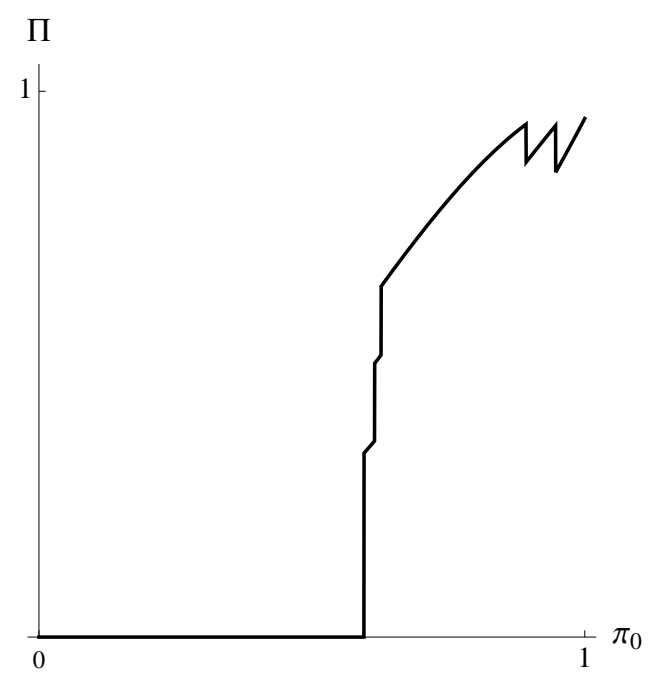

Figure 5: Proposition 5, case ii).

with a fixed timetable, breaks and starting late being impossible. I shall denote the latter as $c$ (commitment job) and the former as $f$ (freelance), and assume that their respective bonuses are $B_{c}$ and $B_{f}$, with $v_{c}$ and $v_{f}$ denoting the corresponding ratios between the opportunity cost of leisure utilities and the bonus. As before the bonus is paid only if the task is completed. With this dual job market, the probability of completion, $\Pi^{D}\left(v_{f}, v_{c} ; \pi_{0}\right)$, will be

$$
\Pi^{D}\left(v_{f}, v_{c} ; \pi_{0}\right)=\left\{\begin{array}{cc}
\Pi\left(\pi_{0}, \alpha, v_{f}\right) & \text { if freelancer } \\
\Pi^{w}\left(\boldsymbol{\pi}\left(\pi_{0}, \alpha\right)\right) & \text { if commitment job. }
\end{array}\right.
$$

The principal would like those agents who should take breaks to be freelancers and the others to choose the commitment job.

The expected utility of an agent who takes the commitment job is equal to

$$
\Pi^{w}(\boldsymbol{\pi}) B_{c}-\left(2-2 \pi_{0} \pi_{1}+\pi_{0}+\pi_{1}\right) e+\left(\pi_{0} \pi_{1}+\left(1-\pi_{0}\right)\left(1-\pi_{1}\right)\right) u .
$$

I shall consider different free-lance bonuses: i) the smallest bonus that satisfies the freelancers participation constraint, i.e, the bonus that makes freelancers indifferent between taking the job or not, and ii) bonuses that induces (some) freelancers not to give up.

The smallest free-lance bonus, $B_{f}$, which will make a freelancer willing to work solves

$$
-e+\pi_{0}(u-e)+\left(1-\pi_{0}\right) 2 u+\pi_{0}^{2} B_{f}=3 u .
$$

Solving for $B_{f}$ leads to

$$
B_{f}=(u+e) \frac{1+\pi_{0}}{\pi_{0}^{2}} \equiv \underline{B}_{f}\left(\pi_{0}\right)
$$


With this bonus all freelancers will give up if they fail in the morning. The commitment job is preferred whenever $B_{c}$ is such that (39) is larger than (40), namely when

$$
B_{c} \geq(u+e) \frac{2-2 \pi_{0} \pi_{1}+\pi_{0}+\pi_{1}}{\Pi^{w}(\boldsymbol{\pi})},
$$

which, after substituting (1) in, can be written as

$$
B_{c} \geq(u+e) \frac{2-2 \pi_{0}^{2} \alpha+\pi_{0}+\pi_{0} \alpha}{\Pi^{w}\left(\boldsymbol{\pi}\left(\pi_{0}, \alpha\right)\right)} \equiv B_{c}\left(\pi_{0}, \alpha\right) .
$$

Let $\tilde{\alpha}$ be the fatigue of the agent the principal wants to be indifferent between the free-lance and the commitment job. By setting $B_{c}=B_{c}\left(\pi_{0}, \tilde{\alpha}\right)$ all agents with $\alpha \leq \tilde{\alpha}$ will choose the free-lance job and all the other agents will prefer the commitment job. The probability of completion is,

$$
\Pi^{D}\left(\underline{v}_{f}\left(\pi_{0}\right), v_{c}\left(\pi_{0}, \tilde{\alpha}\right) ; \pi_{0}\right)=\left\{\begin{array}{cc}
\pi_{0}^{2} & \text { if } \alpha<\tilde{\alpha} \\
\Pi^{w}\left(\boldsymbol{\pi}\left(\pi_{0}, \alpha\right)\right) & \text { if } \alpha \geq \tilde{\alpha}
\end{array}\right.
$$

Note that if $B_{f}<\underline{B}_{f}\left(\pi_{0}\right)$, all freelancers would prefer not to work on the project, yet all agents with $\alpha \geq \tilde{\alpha}$ will choose the commitment job.

Example: $\boldsymbol{\pi}\left(\pi_{0}, \alpha\right)=\pi_{0}\left(1, \alpha, \alpha^{2}\right),(u+e)=1$. The upper side in Figure 6 shows the probability of completion and the bottom side the payments. The increasing thin line is $\Pi^{w}\left(\boldsymbol{\pi}\left(\pi_{0}, \alpha\right)\right)$, the dotted $\Pi^{b}\left(\boldsymbol{\pi}\left(\pi_{0}, \alpha\right)\right)$. The upper envelope of these two lines defines the maximum probability of completion. The values $\hat{\alpha}$ and $\alpha^{*}$ solve $\Pi^{w}\left(\boldsymbol{\pi}\left(\pi_{0}, \alpha\right)\right)=\pi_{0}^{2}$ and $\Pi^{w}\left(\boldsymbol{\pi}\left(\pi_{0}, \alpha\right)\right)=\Pi^{b}\left(\boldsymbol{\pi}\left(\pi_{0}, \alpha\right)\right)$, respectively.

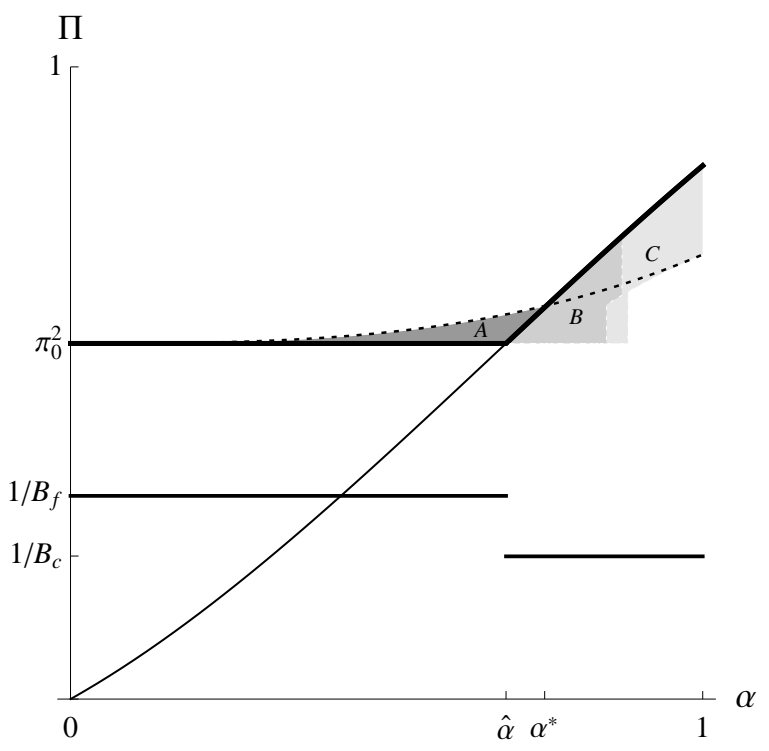

Figure 6: Sorting workers 
Choosing $B_{c}=B_{c}\left(\pi_{0}, \hat{\alpha}\right)$ minimizes the probability loss, relative to the maximum. In this case the loss is equal to the area $A$. Any other cut-off value of $\alpha$ will lead to a larger loss since the freelancers probability of completion is on the $\pi_{0}^{2}$-line and the other agents' on the $\Pi^{w}\left(\boldsymbol{\pi}\left(\pi_{0}, \alpha\right)\right)$-line. Area $A+B$ would be the loss of probability if all workers were free to choose their timetable and faced a single bonus equal to $B_{c}$. Area $A+B+C$ would be the loss if the single bonus $B$ were the lower bonus $B_{f}$, again under free choice of timetable.

Under this two jobs scheme all freelancers take breaks and give up in case of an unsuccessful morning, the probability of completion being independent of $\alpha$ and equal to $\pi_{0}^{2}$. Those who choose the commitment job finish with a probability equal to $\Pi^{w}\left(\boldsymbol{\pi}\left(\pi_{0}, \alpha\right)\right)$. The overall performance is improved (relative to the one with a unique bonus). In particular it is worth noting that this is true also if the common bonus is the (high) commitment payment $B_{c}$. The introduction of two types of jobs leads not only to a higher probability of completion (gain equal to area B) but also to lower expected payment since those agents below $\hat{\alpha}$ are now paid less.

With a high enough free-lance bonus some of the freelancers may prefer not to give up. The bonus $B_{f}$ should be at least

$$
(u+e) \frac{1+\pi_{0} \alpha\left(\pi_{0}\right)}{\pi_{0}^{2} \alpha\left(\pi_{0}\right) f\left(\alpha\left(\pi_{0}\right)\right)} \equiv \hat{B}_{f}\left(\pi_{0}\right)
$$

where $\alpha\left(\pi_{0}\right)$ solves $\Pi^{w}\left(\boldsymbol{\pi}\left(\pi_{0}, \alpha\right)\right)=\Pi^{b}\left(\boldsymbol{\pi}\left(\pi_{0}, \alpha\right)\right)$. $\hat{B}_{f}\left(\pi_{0}\right)$ is the value that would make the individual with fatigue $\alpha\left(\pi_{0}\right)$ indifferent between giving up or not. Note that such an agent is the one who gets less tired among the freelancers and the "cheapest" to persuade not to give up (lemma 3).

Consider a free-lance bonus $B_{f} \geq \hat{B}_{f}\left(\pi_{0}\right)$ so that the agent with $\alpha=\alpha\left(\pi_{0}\right)$ does not want to give up. The principal should set a commitment bonus, $B_{c}$, such that this agent is indifferent between the free-lance and the commitment job:

$$
\Pi^{w}\left(\boldsymbol{\pi}\left(\pi_{0}, \alpha\left(\pi_{0}\right)\right)\right) B_{c}=\Pi^{b}\left(\boldsymbol{\pi}\left(\pi_{0}, \alpha\left(\pi_{0}\right)\right)\right) B_{f}+(u+e) \pi_{0}\left(1-\pi_{0} \alpha\left(\pi_{0}\right)\right) .
$$

Solving for $B_{c}$, and using the fact that $\Pi^{w}\left(\boldsymbol{\pi}\left(\pi_{0}, \alpha\left(\pi_{0}\right)\right)\right)=\Pi^{b}\left(\boldsymbol{\pi}\left(\pi_{0}, \alpha\left(\pi_{0}\right)\right)\right)$, leads to

$$
B_{c}=B_{f}+(u+e) \frac{\pi_{0}\left(1-\pi_{0} \alpha\left(\pi_{0}\right)\right)}{\Pi^{w}\left(\boldsymbol{\pi}\left(\pi_{0}, \alpha\left(\pi_{0}\right)\right)\right)} \equiv B_{c}\left(B_{f}, \pi_{0}\right) .
$$

The following figure illustrates the result above for a $B_{f}>\hat{B}_{f}\left(\pi_{0}\right)$. All agents with $\alpha<$ $\alpha^{*}=\alpha\left(\pi_{0}\right)$ choose the free-lance job, all take a break in the afternoon but some of them do not give up (are on the dotted line). All other workers work all the time. The gain in probability of completion is equal to the area $A_{1}$.

Giving enough incentives to the freelancers not to give up can be very costly. To see this note that the schedules $\left(\underline{B}_{f}\left(\pi_{0}\right), B_{c}\left(\pi_{0}, \alpha\left(\pi_{0}\right)\right)\right)$ and $\left(\hat{B}_{f}\left(\pi_{0}\right), B_{c}\left(\hat{B}_{f}\left(\pi_{0}\right), \pi_{0}\right)\right.$ lead to the same sorting:

$$
\Pi^{D}\left(\underline{v}_{f}\left(\pi_{0}\right), v_{c}\left(\pi_{0}, \tilde{\alpha}\right) ; \pi_{0}\right)=\Pi^{D}\left(\hat{v}_{f}\left(\pi_{0}\right), v_{c}\left(\hat{v}_{f}\left(\pi_{0}\right), \pi_{0}\right) ; \pi_{0}\right)
$$




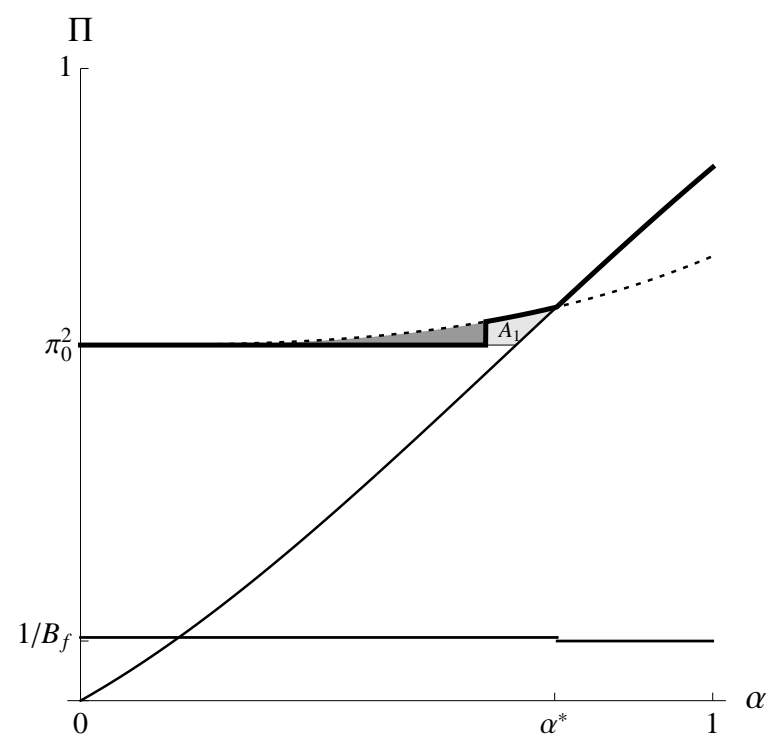

Figure 7: Motivating freelancers

even though

$$
B_{c}\left(\hat{B}_{f}\left(\pi_{0}\right), \pi_{0}\right)>\hat{B}_{f}\left(\pi_{0}\right)>B_{c}\left(\pi_{0}, \alpha\left(\pi_{0}\right)\right)>\underline{B}_{f}\left(\pi_{0}\right)
$$

for all $\pi_{0}<1$. For these payments all agents with $\alpha<\alpha\left(\pi_{0}\right)$ choose the free-lance job and give up; all others work all the time. In order to calculate the optimal payments structure we would need to know the distribution of fatigue in the population, as well as the principal's valuation of the task.

\section{Conclusion}

I have developed a simple three-period principal agent model to analyze the effect of fatigue and innate ability on performance. Fatigue reduces productivity but can be eliminated by taking breaks. If agents differ in fatigue, some benefit from breaks more than others. I show that not all workers should be allowed to take breaks, some should be encouraged. If all workers are given the same contract, specifying a payment and a work timetable, there will always be workers who perform sub-optimally. If all workers are paid the same but can freely choose their own working schedule, there will also be efficiency losses unless leisure/work have no intrinsic utility. Only in this case will all workers behave as if they maximized the probability of completing.

I show that employers can eliminate inefficiencies by introducing a dual job market with a better paid job but which requires working all the time (commitment job) and a lower paid one with the worker having freedom to choose when to work (freelance). I show that workers will sort themselves out in the right way, so that those who should take breaks become freelancers and all the others choose the commitment job. This results give a rationale to having workers 
who work from home. This can lead to increasing productivity and also to lower costs. More so in a world in which many workers commute long distances and office space is a scarce resource.

\section{References}

Florence, P. S. (1924): Economics of Fatigue and Unrest. Routledge.

Hamermesh, D. S. (1990): "Shirking or Productive Schmoozing: Wages and the Allocation of Time at Work," Industrial and Labor Relations Review, 43(3), 121S-133S.

Mednick, S., And M. Ehrman (2007): Take a Nap! Change your life. Workman Publishing.

Mednick, S. C., K. Nakayama, J. L. Cantero, M. Atienza, A. A. Levin, N. Pathak, AND R. Stickgold (2002): "The restorative effect of naps on perceptual deterioration," Nature Neuroscience, 5(7), 677-681.

Ricci, J. A., E. Chee, A. L. Lorandeau, and J. Berger (2007): "Fatigue in the U.S. Workforce: Prevalence and Implications for Lost Productive Work Time," Journal of Occupational and Environmental Medicine, 49(1), 1-10.

Shapiro, C., And J. E. Stiglitz (1984): "Equilibrium unemployment as a worker discipline device," American Economic Review, 74(3), 433-444. 